\title{
SPECTRUM OF COMPLICATED DENGUE FEVER IN RAWAL INSTITUTE OF HEALTH SCIENCES ISLAMABAD: A CROSS SECTIONAL STUDY
}

\author{
Khwaja Ashfaq Ahmed ${ }^{a}$, Nadia Shams ${ }^{a}$, Muhammad Asif Naseer ${ }^{\text {}}$, Hamayun Manzoor Ahmad ${ }^{\text {c }}$ \\ ${ }^{a}$ Associate Professor Department of Medicine, Rawal Institute of Health Sciences, Islamabad. \\ ${ }^{b}$ Head Department of Medicine, Pakistan Air Force Hospital Mushaf. \\ 'House Officer Department of Medicine, Rawal Institute of Health Sciences, Islamabad.
}

\begin{abstract}
:
BACKGROUND \& OBJECTIVE: Pakistan has been facing frequent dengue fever epidemics for the last decade. The current study aims at various complications of dengue fever and their association with age, gender, serology, duration, and symptoms.

METHODOLOGY: This cross-sectional study was conducted at the Medicine department, RIHS Islamabad, over eight months of duration after ethical approval. The adult indoor dengue fever cases were selected by consecutive sampling and informed consent obtained. Cases with malaria co-infection, hematological disease, and severe systemic illness were excluded. The symptoms, hematological counts, serology (dengue NS-1, IgG, and IgM), liver function tests, coagulation profile, pneumonia, and pericardial effusion, were documented. Data were analyzed by SPSS V-22.

RESULTS: Among 75 cases, 19(25\%) were females and 56(75\%) males. The mean age was $33.5 \pm 12.7$. The mean duration of symptoms was $5.4 \pm 2.0$ days. Dengue NS-1 was positive in $45(60 \%)$, IgM in 22(29\%), and IgG in 8(11\%). Symptoms observed were fever $97 \%$, headache $53 \%$, pain abdomen $35 \%$, vomiting $48 \%$, rash $17 \%$, and body aches $39 \%$. Complicated Dengue fever was seen in 21(28\%). Complications include gum bleed $12 \%$, liver impairment $6.6 \%$, gastrointestinal bleed $5 \%$, per-vaginal bleed $4 \%$, epistaxis $4 \%$, hematuria $3 \%$; pleural effusion, ascites and pneumonia $2.6 \%$ each, pericardial effusion $1.3 \%$ and one expiry. Age, gender, dengue serology, and hematological profile wasn't found to be associated with complications.

CONCLUSION: Complications are frequent in indoor dengue cases that include hemorrhagic complications, liver impairment, effusions, and pneumonia. Early presentation to the hospital may be helpful to screen for impending complications with improved morbidity and better patient care.

KEYWORDS: Dengue Fever, Dengue Hemorrhagic Fever, Dengue Shock Syndrome, Capillary Leak Syndrome.
\end{abstract}

How to cite this:

doi: https://doi.org/10.37723/jumdc.v11i4.476

Ahmed KA, Shams N, Naseer MA, Ahmad HM. SPECTRUM OF COMPLICATED DENGUE FEVER IN RAWAL INSTITUTE OF HEALTH SCIENCES ISLAMABAD: A CROSS SECTIONAL STUDY. jumdc. $2020 ; 11(4): 25-32$.

doi: https://doi.org/10.37723/jumdc.v11i4.476

This is an Open Access article distributed under the terms of the Creative Commons Attribution License (http://creativecommons.org/licenses/by/4.0), which permits unrestricted use, distribution, and reproduction in any medium provided the original work is properly cited. 


\section{INTRODUCTION:}

Dengue fever is a vector-borne viral disease transmitted by Aedes Aegypti. It has been estimated that 390 million dengue cases are reported annually all over the world ${ }^{[1]}$. The first epidemic of Dengue fever was observed in Pakistan in 1992 in Karachi ${ }^{[2]}$. For the last 5 years, in almost every fall season, epidemics are observed in Punjab, KPK and Sindh and Kashmir areas of Pakistan ${ }^{[3]}$. A variety of symptoms are seen in dengue fever, including high-grade fever, headache, retro-orbital pain, rash, nausea, vomiting, abdominal pain, bleeding, hypotension, and shock ${ }^{[4]}$.

The variants of dengue fever include classic dengue fever (DF), dengue hemorrhagic fever (DHF), and dengue shock syndrome (DSS). The clinical course, morbidity, and mortality vary among these. The mortality of dengue fever varies from $12-44 \%$. The dengue hemorrhagic fever has a mortality of $2-5 \%$; however, if left untreated, mortality rises up to $50 \%$. The atypical presentations, co-morbid conditions, coagulopathy, superadded infections, geriatrics, pregnancy, malnutrition, and lack of medical care are associated with mortality.

In addition to DHF and DSS, a variety of complications are observed in dengue fever, including cardiovascular (i.e., arrhythmias, myocarditis, pericarditis), central nervous system (encephalitis, meningitis, intracranial hemorrhages, myelitis, Acute demyelinating encephalomyelitis ADEM, neuropathies, GBS, rhabdomyolysis), gastrointestinal and liver (GI hemorrhage, acute liver failure, acalculous cholecystitis, pancreatitis), hematological (thrombocytopenia, pancytopenia), respiratory (ARDS), renal (acute kidney injury, acute tubular necrosis, IgA nephropathy, nephrotic syndrome), ocular (iritis, sub-conjunctival hemorrhages), and genitourinary (orchitis, oophoritis) $)^{[5]}$.

The current study aims to evaluate the frequency of various complications observed in the dengue cases and the possible associations with demographic features, duration of symptoms, serology, and symptoms. The results will be helpful to compare data with regional and international studies. The spectrum of these complications with respect to particular age groups, gender, and other

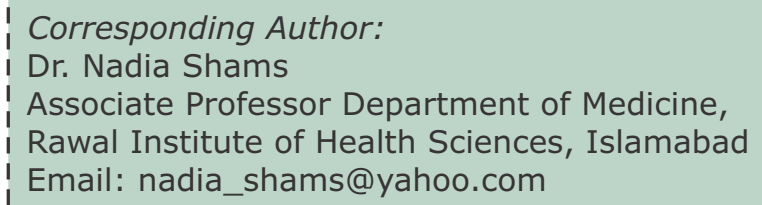

variables will be helpful to identify high-risk cases and reduce morbidity and mortality in our dengue patients.

\section{METHODOLOGY:}

This cross-sectional study was conducted at the Medicine department Rawal Institute of Health Sciences Islamabad (RIHS) from $1^{\text {st }}$ June 2019 to $31^{\text {st }}$ January 2020 after approval from the Research and ethics committee of RIHS (Ref \# RIHS-REC/052/20). Dengue fever was defined as a clinically compatible case of dengue-like illness, dengue, or severe dengue with the confirmatory test, i.e., PCR, antigen, or antibody. DHF was defined as thrombocytopenia (platelets $\leq 100,000 / \mathrm{mm} 3$ and hematocrit $>20 \%$ along with at least two of the following; acute onset of fever for 2-7 days, spontaneous hemorrhagic manifestations or positive tourniquet test, hepatomegaly, or circulatory failure. DSS was defined as the presence of these features along with tachycardia, hypotension, pulse pressure < $20 \mathrm{mmHg}$, cold, clammy skin, or restlessness $[4,6]$. The following patients were included adult patients ( $>18$ years age) fulfilling the clinical and serological criteria for dengue fever diagnosis. Patients from both genders were also included in this study. Exclusion criteria include patients having co-infection with malaria, preexisting hematological disorder (i.e., aplastic anemia, ITP), chronic liver disease, malignancies, and pre-existing severe systemic illness.

Total of 75 adult indoor confirmed cases of dengue fever was selected by consecutive sampling, and informed consent was obtained. The patient's demographic data, detailed history, and clinical examination were performed. The serology, including Dengue NS1, Dengue IgG, and IgM, were performed. The cases with the positive serological test were included. During the hospital stay, their symptoms, clinical findings, hematological 
counts, liver function tests, and coagulation profile were documented. On the basis of clinical findings, electrocardiography, echocardiography, ultrasound abdomen, and other relevant investigations were performed. Patients were specifically examined and investigated for complications like hemorrhage, pneumonia, and pericardial effusion. Data were documented on a specially designed proforma.

Data were analyzed via SPSS version 22. Mean and standard deviation was calculated for quantitative variables (age, duration of symptoms, hematological parameters), frequencies and percentages were calculated for qualitative variables (i.e., gender, dengue serology, and complications observed). A Chisquare test was used to study the association between complicated dengue fever and various variables. Data was presented in the form of a table and pie chart.

\section{RESULTS:}

Among 75 cases of dengue fever, there were $19(25 \%)$ females and $56(75 \%)$ males. Mean age was $33.5 \pm 12.7$ (range $19-65$ years). The mean duration of symptoms was $5.4 \pm 2.0$ days. Dengue NS-1 was positive in $45(60 \%)$, Dengue IgM in 22 (29\%), and Dengue IgG in 8 $(11 \%)$.

Dengue fever without complications was seen in $54(72 \%)$ and with complications in $21(28 \%)$. Gender and age weren't found to be associated with complicated dengue fever $(p>0.05)$. Duration of symptoms was found to be associated with complicated dengue fever, i.e., $6.7 \pm 1.39$ days duration in complicated dengue fever vs. $5.0 \pm 2.04$ days duration in uncomplicated dengue fever cases ( $p$-value 0.018 , table-I).

Commonly observed symptoms were fever

Table-I: The demographic, hematologic, serological variables, and symptomatology of dengue fever cases $(n=75)$.

\begin{tabular}{|c|c|c|c|c|}
\hline Variables & $\begin{array}{l}\text { Among all } \\
(n=75)\end{array}$ & $\begin{array}{l}\text { Un-complicated } \\
\text { dengue fever } \\
(n=54)\end{array}$ & $\begin{array}{c}\text { Complicated } \\
\text { dengue fever } \\
(n=21)\end{array}$ & p-value \\
\hline Age (mean \pm SD) & $\begin{array}{c}33.5 \pm 12.7 \\
(19-65 \text { years })\end{array}$ & $34.19 \pm 13.65$ & $31.86 \pm 10.01$ & 0.512 \\
\hline $\begin{array}{l}\text { Gender } \\
\text { - } \quad \text { Female } \\
\text { - } \quad \text { Male } \\
\end{array}$ & $\begin{array}{l}19(25 \%) \\
56(75 \%)\end{array}$ & $\begin{array}{l}14(73.7 \%) \\
40(71.4 \%)\end{array}$ & $\begin{array}{l}05(26.3 \%) \\
16(28.6 \%)\end{array}$ & 0.850 \\
\hline $\begin{array}{l}\text { Symptoms } \\
\text { Duration }\end{array}$ & $\begin{array}{c}5.45 \pm 2.02 \\
(1-13 \text { days })\end{array}$ & $5.0 \pm 2.04$ & $6.7 \pm 1.39$ & 0.018 \\
\hline $\begin{array}{l}\text { Hematological } \\
\text { profile } \\
\text { - Hemoglobin } \\
\text { - WBC count } \\
\text { - Platelets }\end{array}$ & $\begin{array}{c}11.8 \pm 2.09 \\
4.9 \pm 2.44 \\
104,000 \pm 66226\end{array}$ & $\begin{array}{c}11.88 \pm 2.12 \\
5.18 \pm 2.53 \\
110,000 \pm \\
66,451\end{array}$ & $\begin{array}{c}11.84 \pm 2.06 \\
4.34 \pm 2.11 \\
88,500 \pm \\
63,608\end{array}$ & $\begin{array}{l}0.344 \\
0.790 \\
0.448\end{array}$ \\
\hline $\begin{array}{l}\text { Dengue Serology } \\
\text { - } \quad \text { NS1 } \\
\text { - } \quad \text { IgM } \\
\text { - IgG }\end{array}$ & $\begin{array}{l}45(60 \%) \\
22(29 \%) \\
08(11 \%)\end{array}$ & $\begin{array}{c}33(73 \%) \\
14(63.6 \%) \\
02(25 \%)\end{array}$ & $\begin{array}{c}12(26.7 \%) \\
8(36.4 \%) \\
06(75 \%)\end{array}$ & $\begin{array}{l}0.753 \\
0.299 \\
0.842\end{array}$ \\
\hline $\begin{array}{l}\text { Symptoms } \\
\text { Fever } \\
\text { Headache } \\
\text { Pain Abdomen } \\
\text { Vomiting } \\
\text { Rash } \\
\text { Body aches }\end{array}$ & $\begin{array}{l}73(97 \%) \\
10(13 \%) \\
27(35 \%) \\
36(48 \%) \\
13(17 \%) \\
25(39 \%)\end{array}$ & $\begin{array}{c}53(72.6 \%) \\
09(90 \%) \\
15(55.5 \%) \\
25(69.4) \\
09(69.2 \%) \\
18(72 \%)\end{array}$ & $\begin{array}{c}20(27.4 \%) \\
01(10 \%) \\
12(44.4 \%) \\
11(30.5) \\
04(30.8 \%) \\
07(28 \%)\end{array}$ & $\begin{array}{l}0.482 \\
0.173 \\
0.011 \\
0.636 \\
0.807 \\
1.000\end{array}$ \\
\hline
\end{tabular}

(test of significance Chi-square test; significant $p<0.05$ ) 
$97 \%$, headache $53 \%$, pain abdomen $35 \%$, vomiting $48 \%$, rash $17 \%$, and body aches $39 \%$. Amongst the complications observed, dengue hemorrhagic fever was most frequent, i.e., in $22.6 \%$ cases. Various bleeds observed included gum bleed in $12 \%$, gastrointestinal bleed $5 \%$, per vaginal bleed $4 \%$, epistaxis $4 \%$, and hematuria in $3 \%$. Dengue shock syndrome was seen in $2.6 \%$, liver impairment in $6.6 \%$, pleural effusion, ascites, and pneumonia in $2.6 \%$ each, pericardial effusion in $1.3 \%$, encephalitis in none, and expiry in one case (figure-I). Age, gender, dengue serology, and hematological profile were not found to be associated with complicated dengue fever $(p>0.05)$.

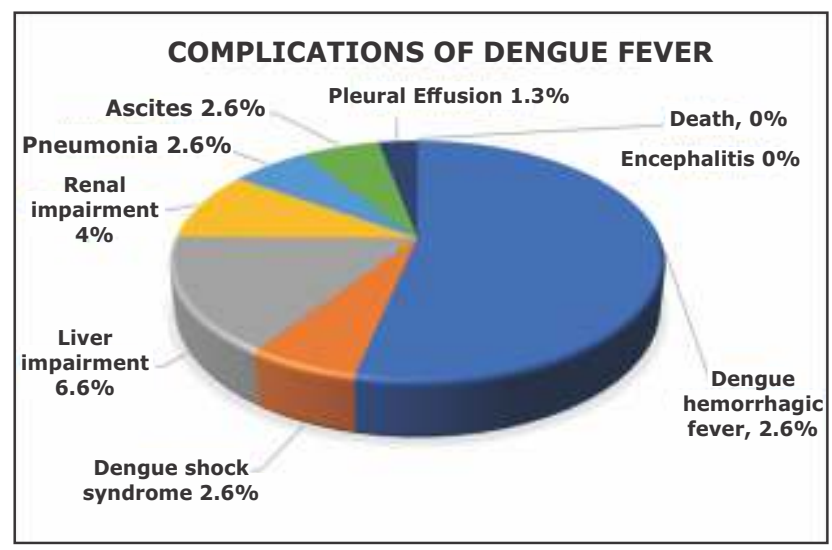

Figure-I: Pie Chart representing frequently observed complications of Dengue Fever $(n=75)$.

\section{DISCUSSION:}

This research was conducted to study the complications observed in indoor dengue fever cases. Twenty-eight percent of the dengue fever cases were observed to have various complications, including hemorrhage, shock, organ involvement, or death, i.e., almost $1 / 3^{\text {rd }}$ of the patients. These figures point towards the need for a protocol to screen the dengue fever cases for complications individually. This screening can be done cost-effectively via history and clinical evaluation. In view of the clinical findings, one can proceed with laboratory or radiological investigations for confirmation. A simplified proforma with a checklist of the parameters to screen the dengue cases for complications may be very useful.
In this study, most of the admitted dengue fever cases were young. The mean age was thirtythree years with few geriatric cases. These results agree with regional studies conducted by Raza et al., ${ }^{[7]}$ and Shams et al., ${ }^{[8]}$ Regarding gender, three fourth of the cases were males with comparatively few female patients. This can be explained by males being frequently involved in outdoor activities in our country as compared to females. Other regional studies also show a predominance of male patients being affected by dengue ${ }^{[7,8]}$. However, the Sri Lankan study by Murugananthan et al. shows almost $50 \%$ female cases ${ }^{[9]}$. Hence, the regional variations in lifestyles and gender-wise outdoor activities justify these results. The gender wasn't related to dengue fever complications in this study. Hence, both the genders to be considered equally prone to complications.

It has been observed that amongst the dengue fever cases filtered in OPD, several stable and uncomplicated cases are managed at home. These patients undergo regular follow up visits, home isolation, and monitoring. Those who require admission are either the DF cases with non-resolving/severe symptoms requiring intravenous medication and monitoring or those with certain complications. Hence criteria for admission isn't dependent absolutely upon the presence of complications, rather upon clinical evaluation and decision by the clinician and the patient preference.

The WHO proposed a criterion for admission of dengue fever cases in 2009. This criterion includes patients with dengue shock (i.e., hypotension), bleeding, the involvement of organs, hematological manifestations (i.e., raised hematocrit), pleural/ pericardial effusions, ascites, gall bladder involvement, comorbid conditions, and social reasons ${ }^{[4]}$. A Sri Lankan study concluded that there was a larger burden of indoor dengue cases on hospitals ${ }^{[10]}$. In response to the WHO recommendations, the admissions and hospital stay of dengue cases was reduced, and it was found to be costeffective as well as safe ${ }^{[4]}$.

The mean duration of symptoms was five days, and the duration at presentation was prolonged in complicated dengue cases as compared to uncomplicated cases ( $p$-value $=0.02)$. A study conducted in Thailand found delayed presentation to hospitals predominantly in 
female cases, though it wasn't linked to complications or mortality ${ }^{[11]}$. Jain et al., found an association between delayed presentation and severity of dengue fever illness ${ }^{[12]}$. Hence, we recommend the early presentation to the hospital in symptomatic cases for the timely screening of complications and decisions regarding the management plan.

The hematological profile in dengue cases showed significant thrombocytopenia with mean platelets counts $104,000 \times 10^{[9]}$. The hemoglobin and white cell counts were not found to be significantly deranged in our dengue cases. In our study, the complicated dengue cases had comparatively lower mean platelets count vs. uncomplicated cases. The severity of thrombocytopenia wasn't found to be associated with complications in dengue fever $(p$-value $=0.20)$. A regional study by Shams et al., found thrombocytopenia in seventy-eight percent cases and leukopenia in forty-nine percent cases ${ }^{[8]}$. Jyanthi et al., recommended that platelet count can be used as a predictor of complications and duration of hospital stay ${ }^{[13]}$. In view of these, it is recommended to screen and follow the hematological profile in dengue cases for timely intervention.

Sixty percent of cases were NS-1 antigenpositive, $29 \%$ were IgM positive that indicates the early phase of dengue, and $11 \%$ were IgG positive at the time of diagnosis. This indicates that the absence of an NS-1 antigen doesn't rule out dengue, and we need to perform antibody tests also depending upon the duration of symptoms. The clinical suspicion and judgment are superior to the laboratory diagnosis during the epidemic season ${ }^{[14]}$. The NS-1 antigen is performed during the first week of illness, and it remains positive till the ninth day; however, serology should be performed after 5 days of symptoms ${ }^{[4,15]}$.

The most frequent symptom in our patients was fever, followed by vomiting that was present in half of the cases and body aches. Abdominal pain was present in $1 / 3^{\text {rd }}$ cases, headache, and rash in < 20\%. CDC reports that the most frequent symptom of dengue includes fever with vomiting, rash, aches, and pains. Regional data agrees with these symptoms demonstrating fever, headache, vomiting, and myalgia being the most frequent symptoms ${ }^{[10]}$. Regarding the complications in our cases, DHF was seen in twenty-two percent of cases. The bleeding manifested as gum bleeding in most of the cases, i.e., $12 \%$. Other hemorrhagic manifestations included a gastrointestinal bleed, hematuria, epistaxis, and per vaginal bleed. This indicates that we have to specifically inquire about the bleeding tendency in every case of dengue fever in view of the fact that many patients ignore minor bleeds.

Dengue shock syndrome (DSS) was seen in two cases. The case fatality of dengue fever is 1$2 \%$, and this rises to $3.5 \%$ for DHF and DSS in Asian countries. Harapan et al., found that there has been a reduction in the case fatality by half in each decade since $1980^{[16]}$.

Liver impairment was seen in seven percent of our cases. Liver involvement in dengue fever presents as hepatomegaly and raised serum transaminases. ${ }^{[17]}$ Sajid et al., observed a variety of symptoms in children with dengue fever, and approximately half of the cases had elevated liver enzymes ${ }^{[18]}$. The spectrum of hepatic involvement may be from asymptomatic raised liver enzymes to liver failure. Hence, it is important to evaluate and screen dengue cases for liver impairment so that hepatotoxic drugs can be avoided, and measures can be taken for recovery of liver functions.

Pleural effusion and ascites were seen in $2.6 \%$ cases each. Ejaz et al., ${ }^{[19]}$ found pleural effusions in sixteen percent of dengue cases. The authors recommend proper clinical examination and chest X-ray in dengue cases for diagnosis of pleural effusion.

Pericardial effusion was seen in one case in this study. Literature shows that cardiac manifestations of dengue fever include pericardial effusion, cardiac tamponade, myocarditis, and elevated cardiac biomarkers ${ }^{[20]}$. Miranda et al., found elevated cardiac biomarkers in $15 \%$ of dengue fever cases and pericardial effusion in $1.2 \%{ }^{[21]}$. We also found pneumonia in $2.6 \%$ of cases. Literature shows that these respiratory complications are seen predominantly in DHF and DSS cases and patients with pre-existing pulmonary conditions ${ }^{[22]}$.

We found neurological manifestations in none of our admitted cases. Literature shows that encephalitis, encephalopathy, meningitis, stroke, cerebellar syndrome, transverse 
myelitis, Guillain Barre' syndrome, myositis, hypokalemic paralysis, and neuritis are found to be associated with dengue ${ }^{[23]}$. Though rare, yet there is a need to create awareness about the neurological manifestations.

There is a wide regional variation in the frequency of complications all over the world. Even the trends of these complications vary in various epidemics across the same region. The results of this study may be able to provide data for comparison with international studies as well as guidance for regional health care workers to diagnose and manage these complications for improved morbidity and prognosis in our patients.

There are certain limitations to this study. We have been unable to perform certain advanced laboratory investigations due to financial constraints. The targeted investigations were performed on the basis of clinical findings that might have underestimated the complications. The authors recommend careful interpretation of study results. There is a need to conduct a multicenter study with an improved sample size focusing on individual complications and their in-depth analysis.

\section{CONCLUSION:}

Varieties of complications are seen in dengue fever, including DHF, DSS, thrombocytopenia, liver impairment, pleural effusion, ascites, and pneumonia. There is a need to screen dengue cases for complications via detailed clinical examination supported by relevant investigations. Early presentation to the hospital may be helpful to screen the cases with impending complications and for improved outcomes.

ACKNOWLEDGEMENT: None.

CONFLICT OF INTEREST: All authors disclose no conflict of interest.

GRANT SUPPORT \& FINANCIAL DISCLOSURES: None.

\section{REFERENCES:}

1. Bhatt S, Gething PW, Brady OJ, Messina JP, Farlow AW, Moyes $\mathrm{CL}$, et al. The global distribution and burden of dengue. Nature. 2013;496(7446):504-507. Doi:10. $1038 /$ nature 12060

2. Chan YC, Salahuddin NI, Khan J, Tan HC, Seah $\mathrm{CL}$, Li J, et al. Dengue hemorrhagic fever outbreak in Karachi, Pakistan, 1994. Transactions of Royal Society of Tropical Medicine and Hygiene. 1995;89:619-620. Doi: 10.1016/0035-9203(95)90412-3

3. Yousaf MZ, Siddique A, Ashfaq UA, Ali M. Scenario of dengue infection and its control in Pakistan: An update and way forward. Asian Pacific Journal of Tropical Medicine. 2018;11:15-23. Available at [https://www.apjtm.org/text.asp?2018/1 $1 / 1 / 15 / 223529]$.

4. World Health Organization. Special Program for Research, Training in Tropical Diseases, World Health Organization. Department of Control of Neglected Tropical Diseases, World Health Organization. Epidemic, Pandemic Alert. Dengue: guidelines for diagnosis, treatment, prevention and control. World Health Organization; 2009.

5. Rajapakse S, Wattegama M, Weeratunga $P$, Sigera PC, Fernando SD. Beyond thrombocytopenia, hemorrhage and shock: the expanded dengue syndrome. Pathogens and Global Health. 2018;112(8):404-414. Doi:10.1080/ 20477724.2018.1552645.

6. Karyanti MR, Uiterwaal CS, Kusriastuti R, Hadinegoro SR, Rovers MM, Heesterbeek $\mathrm{H}$, et al. The changing incidence of dengue hemorrhagic fever in Indonesia: a 45-year registry-based analysis. BMC Infectious Diseases. 2014;14:412. Doi:10.1186/ 1471-2334-14-412.

7. Raza FA, Ur Rehman S, Khalid R, Ahmad J, Ashraf S, Iqbal M, et al. Demographic and 
clinico-epidemiological features of dengue fever in Faisalabad, Pakistan. PLoS One. 2014;9(3):e89868. Doi:10.1371/ journal. pone. 0089868

8. Shams N, Amjad S, Yousaf N, Ahmed W, Seetlani NK, Qaiser N, et al. Predictors of Severity of Dengue Fever in Tertiary Care Hospitals. Journal of Liaquat University of Medical and Health Sciences. 2016;15(4):168-173. Doi:10.22442/ jlumhs. 161540487

9. Murugananthan K, Kandasamy M, Rajeshkannan N, Noordeen F. Demographic and clinical features of suspected dengue and dengue hemorrhagic fever in the Northern Province of Sri Lanka, a region afflicted by an internal conflict for more than 30 years - a retrospective analysis. International Journal of Infectious Diseases. 2014;27:32-36. Doi:10.1016/j.ijid. 2014.04.014

10. Lee LK, Earnest A, Carrasco LR, Thein TL, Gan VC, Lee VJ, et al. Safety and cost savings of reducing adult dengue hospitalization in a tertiary care hospital in Singapore. Transactions of the Royal Society of Tropical Medicine and Hygiene. 2013;107(1):37-42. Doi:10.1093/ trstmh/trs009

11. Wongchidwan N, Wattanagoon Y, Luvira V, Lamsirithaworn S. Delayed care-seeking and outcome of dengue-infected patients. Tropical Doctor. 2018;48:30-33. Doi: $10.1177 / 0049475517712889$

12. Jain S, Mittal A, Sharma SK, Upadhyay AD, Pandey RM, Sinha S, et al. Predictors of Dengue-Related Mortality and Disease Severity in a Tertiary Care Center in North India. Open Forum Infectious diseases. 2017;4(2); ofx056. Doi:10.1093/ofid/ ofx056.

13. Jayanthi HK, Tulasi SK. Correlation study between platelet count, leukocyte count, non-hemorrhagic complications, and duration of hospital stay in dengue fever with thrombocytopenia. Journal of Family
Medicine and Primary Care. 2016;5(1):120-123. Doi:10.4103/22494863.184635

14. Geleta EN. Serological evidence of dengue fever and its associated factors in health facilities in the Borena Zone, South Ethiopia. Research and reports in tropical medicine. $2019 ; 10: 129-136$ Doi: $10.2147 /$ RRTM.S218586

15. Chan $\mathrm{H}$, How $\mathrm{CH}, \mathrm{Ng} \mathrm{CW}$. Definitive tests for dengue fever: when and which should I use? Singapore Medical Journal. 2017; 58(11):632-635. Doi:10.11622/ smedj. 2017100

16. Harapan H, Michie A, Mudatsir M, Sasmono RT, Imrie A. Epidemiology of dengue hemorrhagic fever in Indonesia: analysis of five decades data from the National Disease Surveillance. BMC research notes. 2019;12(1):350. Doi: 10.1186/s13104019-4379-9

17. Kularatne SA, Ralapanawa U, Dalugama C, Jayasinghe J, Rupasinghe S, Kumarihamy P. Series of 10 dengue fever cases with unusual presentations and complications in Sri Lanka: a single centre experience in 2016. BMC Infectious Diseases 2018;18(1):1-8. Doi:10.1186/ s12879018-3596-5.

18. Sajid A, Ikram A, Ahmed M. Dengue Fever Outbreak 2011: Clinical Profile of Children Presenting At Madina Teaching Hospital Faisalabad. Journal of University Medical \& Dental College. 2012; 3(1):42-47.

19. Ejaz K, Khursheed M, Raza A. Pleural effusion in dengue. Saudi Medical Journal. 2011;32(1):46-49.

20. Arora M, Patil RS. Cardiac manifestation in dengue fever. Journal of the Association of Physicians of India. 2016;64(7):40-44.

21. Miranda $\mathrm{CH}$, Borges MD, Matsuno AK, Vilar FC, Gali LG, Volpe GJ, et al. Evaluation of cardiac involvement during dengue viral infection. Clinical Infectious Diseases. 2013;57(6):812-819. Doi:10.1093/cid/ cit403

22. Gupta S, Singh L, Tandon R. Study of pulmonary manifestations among dengue 
patients in tertiary care hospital of North India. International Journal of Contemporary Medical Research. 2020;7(5):E1-E5. Doi:10.21276/ijcmr. 2020.7.5.11.

23. Li GH, Ning ZJ, Liu YM, Li XH. Neurological Manifestations of Dengue infection. Frontiers in Cellular and Infectious Microbiology. 2017; 7:449. Doi: 10.3389/fcimb.2017.00449

\section{Author's contribution:}

Khwaja Ashfaq Ahmed: Study design, data collection, write up, and referencing. Nadia Shams: Data collections and literature review.

Muhammad Asif Naseer: Manuscript write up and review of literature.

Hamayun Manzoor Ahmad: Data collections, data entry, and data analysis.

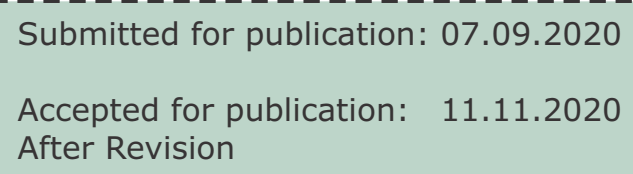

